\title{
Nobiletin prevents TMAO-induced vascular oxidative stress in rats
}

\author{
Guliang Yang ${ }^{\mathrm{a}}$, Chi-Chen Lin ${ }^{\mathrm{b}}$, Li Yuan ${ }^{\mathrm{a}}$, Peilei Wang ${ }^{\mathrm{a}}$, Yiwen Yang ${ }^{\mathrm{a}}$, \\ Xiang Wen ${ }^{c}$, Hui Zhao ${ }^{c^{*}}$, Chi-Tang Ho ${ }^{\mathrm{d}}$ and Shiming $\mathrm{Li}^{\mathrm{a}} \mathrm{d}^{*}$
}

aCollege of Life Sciences, Huanggang Normal University, Huanggang, Hubei, China

bInstitute of Biomedical Science, National Chung-Hsing University, Taichung, Taiwan

${ }^{\mathrm{c}}$ Tianjin Key Laboratory of Food and Biotechnology, School of Biotechnology and Food Science, Tianjin University of Commerce, Tianjin, China ${ }^{\mathrm{d} D e p a r t m e n t}$ of Food Science, Rutgers University, New Brunswick, NJ, USA

${ }^{*}$ Corresponding author: Hui Zhao, Tianjin Key Laboratory of Food and Biotechnology, School of Biotechnology and Food Science, Tianjin University of Commerce, Tianjin, China. E-mail: zhaohui@tjcu.edu.cn; Shiming Li, College of Life Sciences, Huanggang Normal University, Hubei, 438000, China. E-mail: shiming@rutgers.edu

DOI: $10.31665 /$ JFB. 2019.5180

Received: January 10, 2019; Revised received \& accepted: March 15, 2019

Citation: Yang, G., Lin, C.-C., Yuan, L., Wang, P., Yang, Y., Wen, X., Zhao, H., Ho, C.-T., and Li, S. (2019). Nobiletin prevents TMAOinduced vascular oxidative stress in rats. J. Food Bioact. 5: 131-135.

\begin{abstract}
Choline-rich foods in the diet are metabolized by intestinal microflora to trimethylamine (TMA). TMA is subsequently oxidized in the liver by hepatic flavin monooxygenases (FMO), yielding trimethylamine oxide (TMAO) in vivo. TMAO has been reported to cause blood vessel inflammation and induce vascular diseases and atherosclerosis. To investigate the preventive effect of nobiletin on choline chloride-induced vascular oxidative stress and inflammation, the choline chloride-treated rats were intragastric administrated of nobiletin. The serum content of IL-1 $\beta$, MCP-1, IL- 6 and TNF- $\alpha$ were analyzed by Enzyme-Linked Immunosorbent Assay (ELISA), and the contents of glutathione (GSH), malondialdehyde (MDA), nitric oxide (NO) and superoxide dismutase (SOD) were measured. The results revealed that nobiletin decreased the oxidative stress of the aorta and serum inflammatory cytokines level of the experimental rats to reduce the level of vascular inflammation.
\end{abstract}

Keywords: Choline chloride; TMAO; Nobiletin; Oxidative stress; Vascular inflammation.

\section{Introduction}

The association of L-carnitine in high-fat dairy products and egg yolks with high incidence of vascular diseases including atherosclerosis has been convincingly demonstrated in an investigation from over twenty-five hundred people (Koeth, et al., 2013). L-Carnitine is metabolized by intestinal microbiota to release trimethylamine (TMA), which is then absorbed by the gut into portal circulation and rapidly oxidized by hepatic flavin monooxygenases (FMO) in the liver to form trimethylamine oxide (TMAO) and subsequently released to the blood system. TMAO in blood induces oxidative stress and vascular inflammation, attacks vascular cells and causes vascular diseases such as atherosclerosis (He and Chen, 2018; Koeth et al., 2013; Micha et al., 2010). A citrus peel phytochemical, nobiletin $\left(5,6,7,8,3^{\prime}, 4^{\prime}\right.$-hexamethoxyflavone, Nob), was identi- fied as an inhibitor of both nitic oxide (NO) and superoxide $\left(\mathrm{O}_{2}^{-}\right)$ generation in inflammation-associated tumorigenesis (Murakami et al., 2000). It has been found that nobiletin regulates intracellular reactive oxygen species (ROS) generation (Choi et al., 2007), to alternate NF- $\mathrm{KB}$ signaling pathway and decreases the inflammatory markers of COX-2 and iNOS (Xiong et al., 2015). In this study we aimed to investigate whether nobiletin can inhibit TMAO-induced vascular oxidative stress via an anti-inflammation mechanism.

2. Materials and methods

\subsection{Animal experiments}

Forty of Sprague-Dawley (SD) rats, aged 4-6 weeks were pur- 
chased from the Hubei Research Center of Laboratory Animals [Hubei province, China; certification NO. SCXK (E) 2015-0018]. The rats were housed in a room with its temperature maintained at $25 \pm 1{ }^{\circ} \mathrm{C}$ with $50 \%$ relative humidity and $12 \mathrm{~h}$ of light/dark cycle and were with free access to water and normal commercial laboratory rodent diet 5001. All animal experiments were performed in accordance with the Guidelines for the Care and Use of Laboratory Animals, Ministry of Science and Technology, China. Forty $\mathrm{SD}$ rats were randomly divided into five groups (8 rats/group, half male and half female): normal group (NOR), choline chloride-treated group (CON), 100, 200 or $400 \mathrm{mg} / \mathrm{kg}$ bw of nobiletin (98\% purity, purchased from Nanjing Spring \& Autumn Biological Engineering Co., Ltd. (Nanjing, Jiangsu, China) according to the method described [6]) treated group (LCN, MCN, HCN). Except the rats of NOR group, all rats in other groups received drinking water with a mass concentration of $3 \%$ choline chloride (Cat\#: 6748-1, Sinopharm Chemical Reagent Co., Ltd, Shanghai, China) in the entire experimental cycle. All treatments lasted for four weeks after a week of adaptive feeding. Twentyfour hours after the final dose administration, all rats were euthanized by $\mathrm{CO}_{2}$ asphyxiation and laparotomy was performed. Proximal aorta and blood samples were collected from each animal.

\subsection{Histopathological analyses}

The proximal aorta samples were fixed in $10 \%(\mathrm{v} / \mathrm{v})$ neutral formalin for $24 \mathrm{~h}$, and paraffin sections were made according to the routine method. To observe the pathological symptoms of the tissue, Hematoxylin and eosin (H\&E) staining was carried out according to standard protocols. The aortic tissue was sliced and fixed with $10 \%$ formalin, and then stained with oil red O staining. Subsequently, the slices were observed at $200 \times$ magnification under a BH2 optical microscope (Olympus, Hino, Tokyo, Japan).

\subsection{Determination of serum inflammatory cytokines of IL-16, MCP-1, IL-6 and TNF- $\alpha$}

All the experimental rats were euthanized after four weeks consecutive treatment. Blood was collected from three randomly selected rats of each group, and the serum was obtained after centrifugated at $1,500 \mathrm{rpm}$ for $10 \mathrm{~min}$. The serum pro-inflammatory cytokines, (Interleukin-1 $\beta$ (IL-1 $\beta$, abcam, \# ab100768), monocyte chemotactic protein-1 (MCP-1, abcam, \# ab100778), IL-6 (abcam, \# ab100772), and tumor necrosis factor (TNF)- $\alpha$ (abcam, \# ab100785) levels were determined using Enzyme-Linked Immunosorbent Assay (ELISA) according to the manufacturer's instructions. The optical density value at a wavelength of $450 \mathrm{~nm}$ of each well was read immediately using a microtiter plate reader (LabSystems Multiskan MS, Thermo Fisher Scientific Inc., Waltham, MA, USA).

\subsection{Determination of serum GSH-ST, MDA, NO and SOD levels}

Blood samples obtained from rats were naturally coagulated for $20 \mathrm{~min}$ after collection then centrifuged at $112 \times \mathrm{g}$ for $10 \mathrm{~min}$. The supernatant was collected and stored at $-20^{\circ} \mathrm{C}$. Serum GSH-ST (A004), MDA (A003), NO (A012) and SOD (A001) levels were determined using GSH-ST, MDA, NO or SOD detection kit (purchased from Nanjing Jiancheng Bioengineering Institute, Nanjing, China) according to the manufacturer's instructions. Absorbance was measured on a MK3 Microplate reader (Thermo Fisher Scien- tific Inc., Waltham, MA, USA).

\subsection{Statistical analysis}

Statistical evaluate was performed by running the one-way analysis of variance (ANOVA) or one-way Student's t test and Duncan's Multiple Range Test. Data were presented as the means \pm SD for the indicated number ( $\geq 3$ replicates) of independently performed experiments. A probability value of $p<0.01$ was considered statistically significant.

\section{Results}

\subsection{Histological assessment}

It was observed from histological analyses (Figure 1) that choline chloride treated mice suffered from intimal thickening, slight endothelial tissue shedding and local elevating in the aortic intima close to the cardiac. Proliferation of elastic fibers in the tunica media of the aorta was also observed. Vacuolar degeneration was also found in the intima and tunica media, which demonstrates that choline chloride induced a cardiovascular inflammation. The results of oil red O staining (Figure 2) showed that the vessels in the outer and middle layers were stained red in the choline chloridetreated rats' aorta. After co-treatment with nobiletin, the aorta lipids accumulation was significantly reduced, and the inhibition of nobiletin was obvious with dose increase. The histological phenotypes indicated above are consistent with the reported findings, i.e. generated from choline (Seldin et al., 2016; Tang and Hazen, 2014) L-carnitine (Koeth et al., 2013), or phosphatidylcholine (Tang et al., 2013) among others, TMAO induces vascular inflammation (Seldin et al., 2016). Treatment with nobiletin showed that aortic intimal thickening was greatly reduced, the aortic smooth muscle cells arranged evenly, and smooth muscle cell hyperplasia was not observed. Hence, conclusion can be drawn that nobiletin efficiently prevents cardiovascular inflammation induced by choline chloride.

\subsection{Nobiletin regulating the expression of serum IL-16, MCP-1, IL-6 and TNF- $\alpha$}

The serum concentration of IL-1 $\beta$, MCP-1, IL- 6 and TNF- $\alpha$ was measured (Figure 3). The expression levels of IL-1 $\beta$, MCP-1, IL-6 and TNF- $\alpha$ were low in the NOR group, whereas choline treatment (CON group) led to a remarkable up-regulation of the above mentioned four cytokines in the serum. In the meantime, the contents of IL- $1 \beta$, MCP-1, IL- 6 and TNF- $\alpha$ in the serum of the HCN treated group were down-regulated to $38.8 \%, 62.8 \%, 43.1 \%$ and $38.4 \%$, respectively, compared with the $\mathrm{CON}$ group, demonstrating that nobiletin inhibited choline chloride (TMAO) induced inflammation.

\subsection{Effect of nobiletin on serum GSH-ST, MDA, NO and SOD}

To address the oxidative status in choline-exposed mice without or with nobiletin co-treatment, serum levels of GSH-ST, MDA, NO, SOD were examined (Figure 4). The results of this work indicates that choline alone (CON group) markedly enhanced MDA content, inducing a 4.5-fold increase comparing with the NOR group, while dramatically reducing SOD activity to only $34.7 \%$ of NOR group. However, the changes stimulated by choline chloride were dra- 

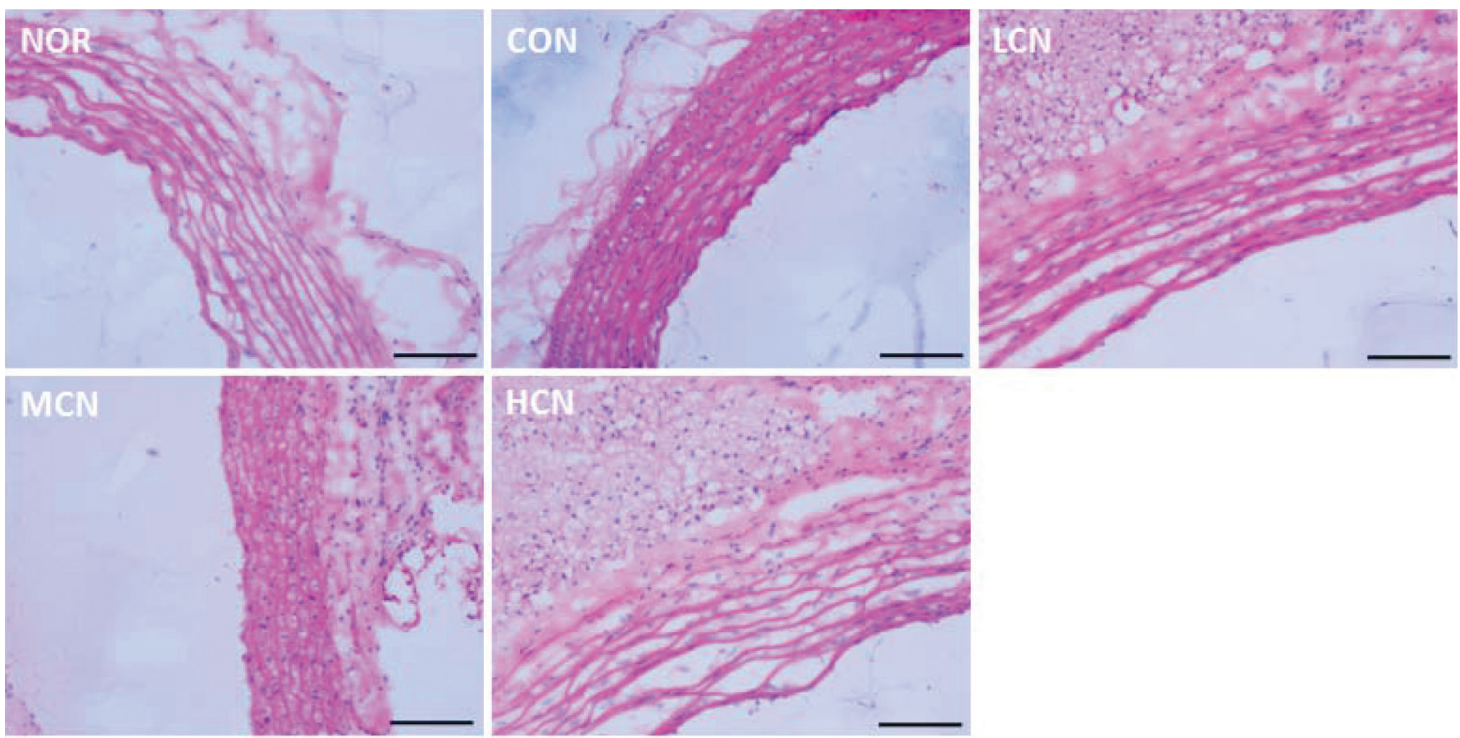

Figure 1. H\&E staining results of cardiovascular tissue in rats of different treatment groups. (At the end of the experiment, the proximal aorta samples from five randomly selected rats in each group were harvested, then H\&E staining was performed. The scale bar represents $200 \mu \mathrm{m}$ )

matically mitigated after nobiletin treatment. It is noteworthy that the elevated MDA levels and decreased SOD activity after choline chloride stimulation revealed that excessive oxidation had occurred in choline chloride induced rat model of cardiovascular inflammation. The findings that nobiletin repressed choline-induced inflammatory response indicates the strong antioxidant capability of nobiletin, in agreement with the results from the antioxidant activity in a yeast model (Wang et al., 2018).

\section{Discussion}

Mounting evidence has shown that western high-fat diets rich in phosphatidylcholine, choline, L-carnitine and other TMA-containing nutrients are closely associated with the development of cardiovascular diseases (CVD), such as atherosclerosis (Brown and Hazen, 2018). Recent advances in the research of microbiota function on TMA-generating foods, such as choline chloride, suggested that the TMAO molecule after FMO oxidation of TMA specifically and rapidly activates endothelial cell-specific signaling events resulting in vascular disease pathogenesis. Additionally, TMAO-enhanced risk of vascular damage is closely associated with the overproduction of both ROS and inflammatory cytokines (Violi et al., 2017). Hence, it can be rationalized based on these findings that targeting the inhibition of TMAO generation in the microbial stage may provide an efficient novel strategy in delay-
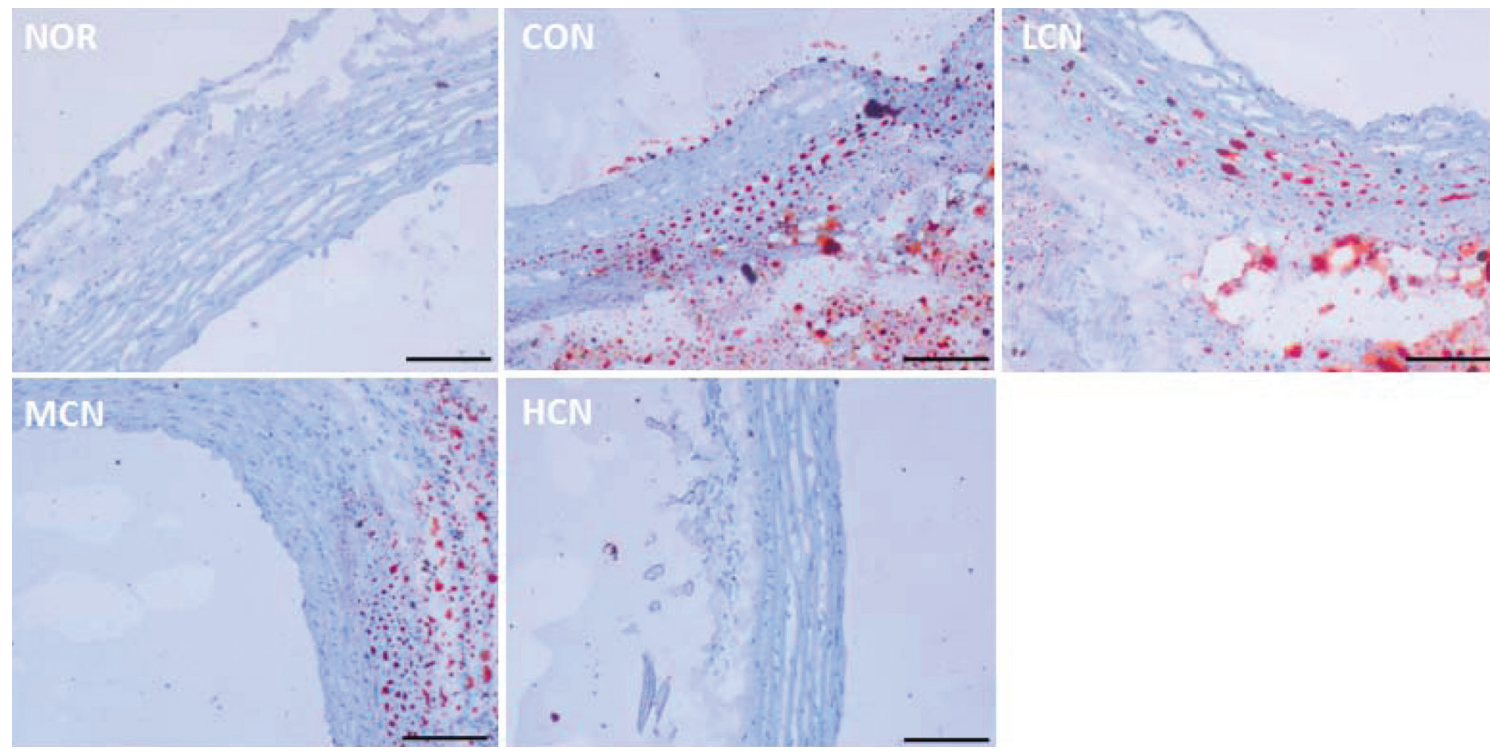

Figure 2. Oil red $\mathbf{O}$ staining results of cardiovascular tissue in rats of different treatment groups. (At the end of the experiment, the proximal aorta samples from five randomly selected rats in each group were harvested, then oil red O staining was performed. The scale bar represents $200 \mu \mathrm{m}$ ) 


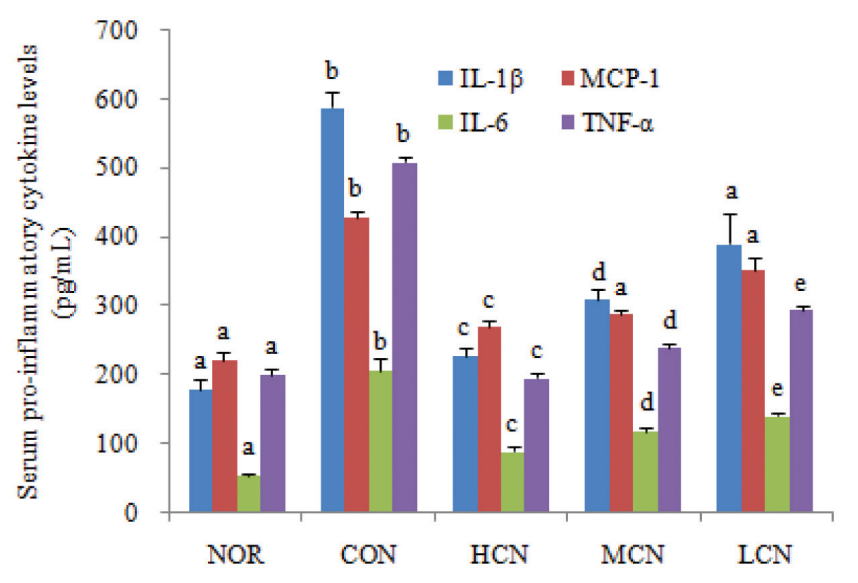

Figure 3. Effect of nobiletin on serum pro-inflammatory cytokines level in choline chloride-treated SD rats. The average content of serum proinflammatory cytokines of each group is expressed as the mean \pm SD ( $=3$ per group). Values not sharing the same superscript letters (a-e) of the same indexs are significantly different between the groups. $p<0.01$.

ing, preventing, even curing vascular diseases by reducing oxidative stress and inhibiting inflammation.

In cardiovascular disease (CVD) prevention, vegetables and fruits are excellent sources of heart-healthy diet due to their rich content of phytochemicals and dietary fiber. A myriad of epidemiological data suggests that intake of dietary phytochemicals, such as polyphenols, have health promoting benefits in reducing vascular diseases through attenuating oxidative stress and inflammation (Otto, 2016). As a dietary medicinal food in China for many centuries, tangerine peel is rich in polyhydroxyflavonoids such as hesperidin, and polymethoxyflavones, such as nobiletin and tangeretin. The mechanism of anti-inflammatory effects of polymethoxyflavones was to inhibit NF- $\kappa \mathrm{B}$ and ROS production, which are the major pathogenic causes of vascular diseases (Mulvihill et al, 2011). Nobiletin was found to effectively prevent atherosclerosis induced by low density lipoproteins at the level of vascular walls by targeting macrophage foam-cell formation and reducing plasma cholesterol concentrations, as well as by inhibiting the oxidation of low density lipoproteins (Mulvihill et al, 2011). In this study, in vivo experiments indicated the preventive effect of nobiletin on choline (TMAO) stimulated cardiovascular inflammation. Furthermore, we identified that the inhibition of ROS-mediated oxidative stress underlie the preventative effect of nobiletin on vascular endothelial cells. Excess ROS plays a key role in the inflammatory pathogenesis. The cellular constitutes including proteins, lipids, and DNA will be oxidized upon cells receiving oxidative stimuli (Manish et al, 2014; Meng et al., 2017). Vascular endothelium usually acts as barrier blocking macromolecules and inflammatory cells from the blood to tissue. The oxidative stress leads to the migration of inflammatory cells through the endothelial barrier and generates carotid artery atheromatous plaque. Previous studies have shown that pro-atherogenic stimuli induce the generation of oxidative stress in vascular endothelial cells and result in intimalmedial thickening and arteriosclerotic progression (Li and Förstermann, 2013). Nobiletin regulated the oxidative markers such as GSH-ST, MDA, NO and SOD, which were altered by TMAO stimulation.

In summary, the results of this study showed that nobiletin reduced the damage of TMAO to the vascular by decreasing the level of ROS-mediated oxidative stress, and inhibiting inflammation.

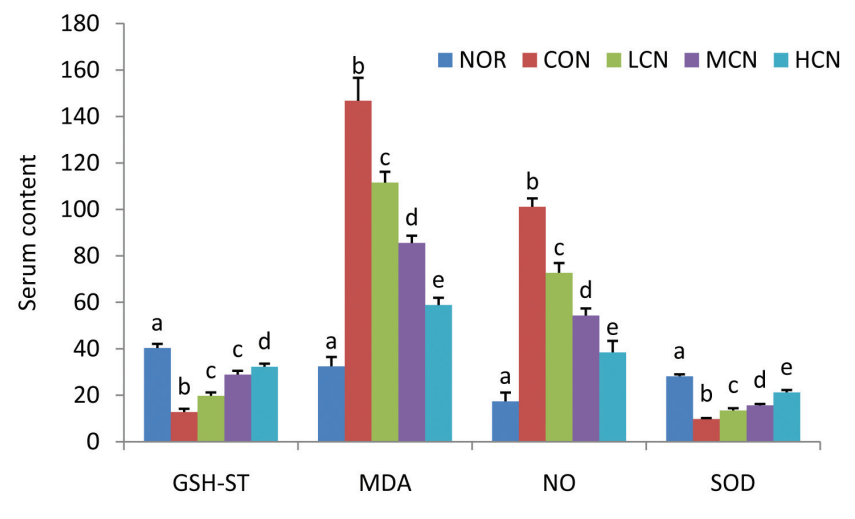

Figure 4. Effects of nobiletin on the content of liver function and oxidative metabolites in vivo. Serum levels of GSH-ST (U/L), MDA (nmol/mL), $\mathrm{NO}(\mathrm{nmol} / \mathrm{mL})$ and SOD $(\mathrm{U} / \mathrm{mL})$. The average content of data in each group is expressed as the mean \pm SD (A: $n=8$ per group). The significant difference among each group was analyzed by one-way ANOVA and Duncan's multiple range tests. Different letters of a-e represent significant differences of the same indexes when $p<0.01$.

\section{Acknowledgments}

Grant support from (1) National Nature Science Fund of China (GRANT number 31571832) (2) Tianjin Innovative Research Team Grant (TD13-5087) and Tianjin Key Laboratory of Food Biotechnology Grant (TJCU-KLFB-18101).

\section{References}

Brown, J.M., and Hazen, S.L. (2018). Microbial modulation of cardiovascular disease. Nat. Rev. Microbiol. 16: 171-181.

Choi, S.Y., Hwang, J.H., Ko, H.C., Park, J.G., and Kim, S.J. (2007). Nobiletin from citrus fruit peel inhibits the DNA-binding activity of NF-kappaB and ROS production in LPS-activated RAW 264.7 cells. J. Ethnopharmacol. 113: 149-55.

Koeth, R.A., Wang, Z., Levison, B.S., Buffa, J.A., Org, E., Sheehy, B.Y., Britt, E.B., Fu, X., Wu, Y., Li, L., Smith, J.D., Didonato, J.A., Chen, J., Li, H., Wu, G.D., Lewis, J.D., Warrier, M., Brown, J.M., Krauss, R.M., Tang, W.H.W., Bushman, F.D., Lusis, A.J., and Hazen, S.L. (2013). Intestinal microbiota metabolism of $L$-carnitine, a nutrient in red meat, promotes atherosclerosis. Nat. Med. 19: 576-585.

He, Z., and Chen, Z.-Y. (2018). The role of trimethylamine-N-oxide (TMAO) and its role in development of atherosclerosis. J. Food Bioact. 2: 28-36.

Manish, M., Rizwan, S.M., Khiem, T., Sekhar, P.R., and Asrar, B.M. (2014). Inflammation and tissue injury. Antioxid. Redox Sign. 20: 1126-1167.

Meng, D., Zhang, P., Li, S., Ho, C-T., and Zhao, H. (2017). Antioxidant activity evaluation of dietary phytochemicals using Saccharomyces cerevisiae as a model. J. Funct. Foods 38: 36-44.

Li, H., Horke, S., and Förstermann, U. (2013). Oxidative stress in vascular disease and its pharmacological prevention. Trends Pharmacol. Sci. 34: 313-319.

Micha, R., Wallace, S.K., and Mozaffarian, D. (2010). Red and processed meat consumption and risk of incident coronary heart disease, stroke, and diabetes mellitus: a systematic review and meta-analysis. Circulation. 121: 2271-2283.

Mulvihill, E.E., Assini, J.M., Lee, J.K., Allister, E.M., Sutherland, B.G., Koppes, J.B., Sawyez, C.G., Edwards, J.Y., Telford, D.E., Charbonneau, A., St-Pierre, P., Marette, A., and Huff, M.W. (2011). Nobiletin attenuates VLDL overproduction, dyslipidemia, and atherosclerosis in mice with diet-induced insulin resistance. Diabetes. 60: 1446-1457.

Murakami, A., Nakamura, Y., Torikai, K., Tanaka, T., Koshiba, T., Koshimizu, K., Kuwahara, S., Takahashi, Y., Ogawa, K., Yano, M., Tokuda, H., Nishi- 
no, H., Mimaki, Y., Sashida, Y., Kitanaka, S., and Ohigashi, H. (2000). Inhibitory effect of citrus nobiletin on phorbol ester-induced skin inflammation, oxidative stress, and tumor promotion in mice. Cancer Res. 60(18): 5059-5066.

Otto, C.M. (2016). Heartbeat: Dietary polyphenols and vascular function. Heart. 102: 1337-1338.

Seldin, M.M., Meng, Y., Qi, H., Zhu, W., Wang, Z., Hazen, S.L., Lusis, A.J., and Shih, D.M. (2016). Trimethylamine N-oxide promotes vascular inflammation through signaling of mitogen-activated protein kinase and nuclear factor-kB. J. Am. Heart Assoc. 5: e002767.

Tang, W.H.W., and Hazen, S.L. (2014). The contributory role of gut microbiota in cardiovascular disease. J. Clin. Invest. 124: 4204-4211.

Tang, W.H.W., Wang, Z., Levison, B., Koeth, R.A., Britt, E.B., Fu, X., Wu, Y., and Hazen, S.L. (2013). Intestinal microbial metabolism of phosphatidylcholine and cardiovascular risk. N. Engl. J. Med. 368: 1575-1584.

Violi, F., Loffredo, L., Carnevale, R., Pignatelli, P., and Pastori, D. (2017) Atherothrombosis and oxidative stress: mechanisms and management in elderly. Antioxid Redox Sign. 27: 1083-1124.

Wang, M., Meng, D., Zhang, P., Wang, X., Du, G., Brennan, C., Li, S., Ho, C-T., and Zhao, H. (2018). Antioxidant protection of nobiletin, 5- demethylnobiletin, tangeretin, and 5 demethyltangeretin from citrus peel in Saccharomyces cerevisiae. J. Agric. Food Chem. 66: 3155-3160.

Xiong, Y., Chen, D., Yu, C., Lv, B., Peng, J., Wang, J., and Lin, Y. (2015). Citrus nobiletin ameliorates experimental colitis by reducing inflammation and restoring impaired intestinal barrier function. Mol. Nutr. Food Res. 59: 829-842. 